\title{
Intolerance to Multiple P2Y12 Inhibitors Following Percutaneous Coronary Intervention
}

\author{
Mina Shnoda ${ }^{1}$, Kinan Kassar ${ }^{2}$, Deanna L. Huffman ${ }^{1}$, Vijaya Sanikommu ${ }^{3}$, Indu Poornima ${ }^{2}$ \\ 1. Internal Medicine, Allegheny Health Network, Pittsburgh, USA 2. Cardiovascular Disease, Allegheny Health \\ Network, Pittsburgh, USA 3. Cardiology, Allegheny Health Network, Pittsburgh, USA
}

Corresponding author: Mina Shnoda, minamohebhalim@gmail.com

\begin{abstract}
Dual antiplatelet therapy (DAPT), defined as administration of a P2Y12 receptor inhibitor (clopidogrel, prasugrel, or ticagrelor) and aspirin, is recommended after percutaneous coronary intervention. We describe a case of a 50-year-old gentleman with intolerance to the three previously mentioned P2Y12 inhibitors following the placement of a drug-eluting stent to the left anterior descending artery. To our knowledge, based on a thorough review of the literature, this is the second case reporting a similar medical dilemma. We have discussed the multidisciplinary approach implemented to overcome this clinical challenge, which involved the use of clopidogrel with simultaneous administration of a six-day course of oral steroids.
\end{abstract}

Categories: Cardiology

Keywords: p2y12 inhibitors, clopidogrel hypersensitivity, multiple drug intolerance, percutaneous coronary intervention

\section{Introduction}

Patients with hypersensitivity to any single P2Y12 inhibitor following percutaneous coronary intervention (PCI) are commonly managed by shifting to a different P2Y12 inhibitor $[1,2]$. We present a case of a post-PCI patient with intolerance to multiple P2Y12 inhibitors and the multi-disciplinary approach used to overcome such a clinical challenge.

Review began 01/22/2021 Review ended 02/21/2021 Published 02/26/2021

\section{(c) Copyright 2021}

Shnoda et al. This is an open access article distributed under the terms of the Creative Commons Attribution License CC-BY 4.0., which permits unrestricted use, distribution, and reproduction in any medium, provided the original author and source are credited.

\section{Case Presentation}

A 50-year-old male with past medical history of ischemic heart disease and prasugrel hypersensitivity in the form of generalized rash underwent elective PCI with one drug-eluting stent (DES) to the left anterior descending artery (LAD). He was subsequently started on dual antiplatelet therapy with aspirin $81 \mathrm{mg}$ daily and ticagrelor $90 \mathrm{mg}$ twice a day.

Two days following discharge, the patient was complaining of severe shortness of breath with minimal exertion, and occasionally at rest. He denied any fevers, chills, productive cough, orthopnea, paroxysmal nocturnal dyspnea or lower limb edema. The patient's dyspnea was heavily investigated with basic labs, cardiac biomarkers, imaging of the chest, and repeat transthoracic echocardiogram. All results came back within normal limits. The patient's dyspnea was deemed secondary to ticagrelor use and the drug was discontinued, with subsequent improvement of his symptoms. Given his history of prasugrel hypersensitivity and current intolerance to ticagrelor, the patient was started on clopidogrel. Three days later, he presented to the emergency department (ED) with a rash that started on his chest and spread to his arms and face (Figure 1).

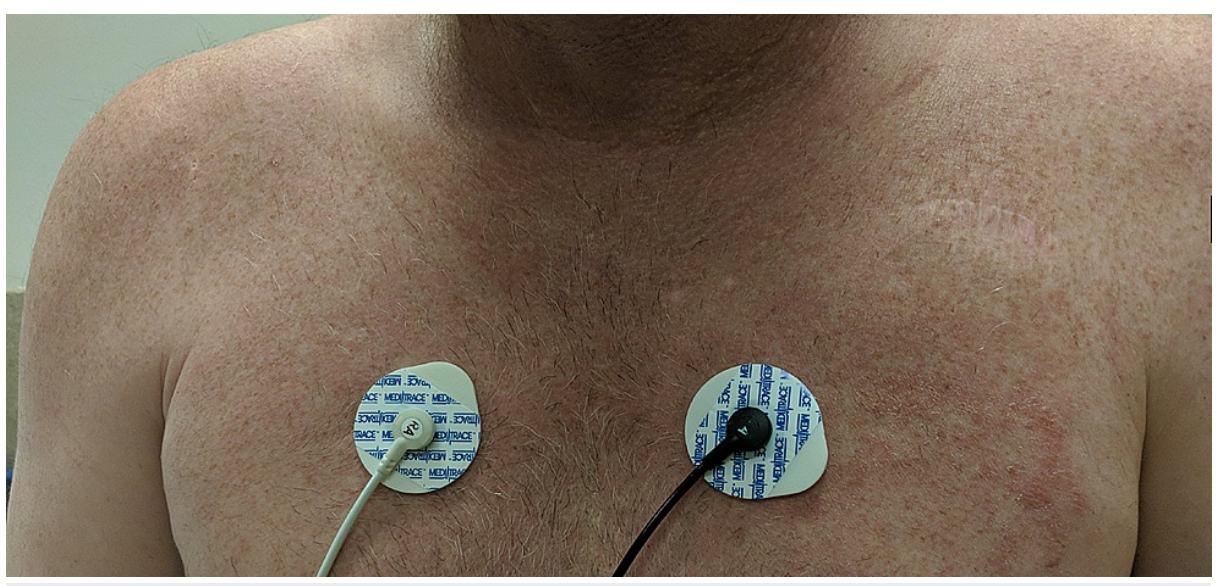

FIGURE 1: Rash predominantly affecting patient's chest 
The patient was diagnosed with cutaneous hypersensitivity to clopidogrel. Given the patient's need for dual antiplatelet therapy and previously documented prasugrel hypersensitivity as well as intolerance to ticagrelor, shifting to any of them was not an option.

Reviewing the literature, three management strategies have been described for clopidogrel hypersensitivity, including switching to another agent, implementing a desensitization protocol, and a six-day course of steroids without clopidogrel discontinuation. A shared decision was made to implement the latter approach however with a slow taper over a three-week period. The patient was started on oral prednisone $30 \mathrm{mg}$ twice a day for five days, followed by a decrease of $5 \mathrm{mg} /$ day every three days for 15 days. On his one-week followup, the patient was well-tolerating clopidogrel with complete resolution of his symptoms and no significant side effects from steroids. The patient showed no side effects related to steroids on his one-month followup.

\section{Discussion}

The current American College of Cardiology Foundation/American Heart Association Task Force on Practice Guidelines/Society for Cardiovascular Angiography and Interventions (ACCF/AHA/SCAI) guidelines recommend at least 12 months of dual antiplatelet therapy after DES implantation. Clopidogrel is widely used after DES placement to protect against stent thrombosis.

Clopidogrel hypersensitivity incidence is estimated at $6 \%$ and results in premature drug discontinuation in $1.5 \%$ of patients [3]. It is believed to be secondary to lymphocyte-mediated delayed hypersensitivity and presents clinically in one of three forms. The first and most common presentation is in the form of a generalized, pruritic rash affecting the trunk with or without the involvement of upper or lower extremities; second, a rash limited to the localized area involving the neck, face, back, axilla, palms, or soles. The third and least common is in the form of angioedema [1]. A skin biopsy was not obtained in our case study as the patient opted against any invasive investigation.

Several management strategies for clopidogrel hypersensitivity have been described in the medical literature, including shifting to another P2Y12 inhibitor agent or clopidogrel desensitization [1,2]. However, there is an increased likelihood of cross-reactivity between clopidogrel, prasugrel, and ticlopidine attributed to their similar thienopyridine structure [4,5], whereas ticagrelor is a nonthienopyridine P2Y12 inhibitor and preferred over other agents for the theoretical less risk for cross-reactivity with clopidogrel [6]. Ticagrelor use is associated with mild-moderate dyspnea in approximately $14 \%$ of patients, with $0.9 \%$ of those requiring discontinuation of the drug [7].

The current case report describes a patient with a recently placed DES presenting with hypersensitivity reaction to clopidogrel. His past medical history was further complicated by documented hypersensitivity to prasugrel and severe dyspnea associated with the use of ticagrelor. Intolerance to the three P2Y12 inhibitors has seldom been reported in the literature [8] and represents a serious management dilemma in post-PCI patients.

\section{Conclusions}

In our case study, clopidogrel hypersensitivity was successfully managed without discontinuation of clopidogrel. The administration of a six-day course of oral steroids resulted in complete resolution of symptoms. The successful management supports the conclusion previously drawn by the study done by Cheema and his colleagues, demonstrating that treatment with a short course of oral steroids can be safe and highly effective in the management of cutaneous clopidogrel hypersensitivity, especially in the absence of alternative solutions.

\section{Additional Information \\ Disclosures}

Human subjects: Consent was obtained or waived by all participants in this study. Conflicts of interest: In compliance with the ICMJE uniform disclosure form, all authors declare the following: Payment/services info: All authors have declared that no financial support was received from any organization for the submitted work. Financial relationships: All authors have declared that they have no financial relationships at present or within the previous three years with any organizations that might have an interest in the submitted work. Other relationships: All authors have declared that there are no other relationships or activities that could appear to have influenced the submitted work.

\section{References}

1. Cheema AN, Mohammad A, Hong T, et al.: Characterization of clopidogrel hypersensitivity reactions and management with oral steroids without clopidogrel discontinuation. J Am Coll Cardiol. 2011, 58:1445-1454. 


\section{Cureus}

10.1016/j.jacc.2011.06.040

2. Lokhandwala J, Best PJM, Henry Y, Berger PB: Allergic reactions to clopidogrel and cross-reactivity to other agents. Curr Allergy Asthma Rep. 2011, 11:52-57. 10.1007/s11882-010-0152-9

3. CAPRIE Steering Committee: A randomised, blinded, trial of clopidogrel versus aspirin in patients at risk of ischaemic events (CAPRIE). Lancet. 1996, 348:1329-1339. 10.1016/s0140-6736(96)09457-3

4. Kim S-H, Park S-D, Baek Y-S, et al.: Prasugrel-induced hypersensitivity skin reaction. Korean Circ J. 2014, 44:355-357. 10.4070/kcj.2014.44.5.355

5. Makkar K, Wilensky RL, Julien MB, Herrmann HC, Spinler SA: Rash with both clopidogrel and ticlopidine in two patients following percutaneous coronary intervention with drug-eluting stents. Ann Pharmacother. 2006, 40:1204-1207. 10.1345/aph.1G587

6. Harris JR, Coons JC: Ticagrelor use in a patient with a documented clopidogrel hypersensitivity . Ann Pharmacother. 2014, 48:1230-1233. 10.1177/1060028014539143

7. Mahaffey KW, Wojdyla DM, Carroll K, et al.: Ticagrelor compared with clopidogrel by geographic region in the Platelet Inhibition and Patient Outcomes (PLATO) trial. Circulation. 2011, 124:544-554.

10.1161/CIRCULATIONAHA.111.047498

8. Chin N, Rangamuwa K, Mariasoosai R, Carnes J, Thien F: Oral antiplatelet agent hypersensitivity and crossreactivity managed by successful desensitisation. Asia Pac Allergy. 2015, 5:51-54.

10.5415/apallergy.2015.5.1.51 